\title{
LA RECHERCHE HYDROLOGIQUE DANS LA CONSERVATION DU SOL
}

\author{
Lloyd L. HARROLD
}

Il est nécessaire, pour de nombreuses raisons, que Je programme du "Service de Conservation du Sol des Etats-Unis » s'appuie sur des recherches expérimentales. Sa tâche est d'établir des projets d'établissements fermiers en vue d'assurer une agriculture durable, la lutte contre les crues, et le meilleur usagc des ressources en eau. Pour cela, il est essentiel de connaitre la réaction des procédés de culture et des caractéristiques du terrain sur la « conservation 》 du sol et des eaux. Il faut peser la valeur des mithodes couramment pratiquées devant chaque aspect de la conservation. On doit conduire les recherches, les expériences et les études, dans le but de développer des principes et des procédés nowveaux et profitables.

Les recherches agricoles sont effectuees pour la plupart, sur des terrains de dimensions restreintes. L'application de méthodes statistiques demande de nombreux recoupements, d'où la nécessité pratique de réduire les dimensions des terrains d'expériences, pour pouvoir les multiplier. Les résultats obtenus quant à la qualité et à l'abondance des récoltes, peuvent généralement s'appliquer à de plus grandes superficies. Par contre, une extension directe n'est plus possible en ce qui concerne les débits de ruissellement et les entrainements du terrain par érosion. C'est pourquoi une station de recherches hydrologiques, faisant partie du programme de conservation des sols des Etats-Unis, a été montrée à Coshocton (Ohio), sur un bassin versant type, comme le montrent les figures 1 et 2 . C'est une des multiples stations de recherche de conservation du sol installées dans diverses régions des Etats-Unis. Tous les travaux de recherche du service de conservation du sol sont d'ailleurs faites en collaboration avec les diverses stations expérimentales d'Etat.

Voici la nature des études faites sur ces bassins expérimentaux :

$1^{\circ}$ Déterminer quantitativement l'effet des méthodes perfectionnées d'utilisation du sol et de lutte contre l'érosion sur la conservation du sol et des caux. Evaluer dans quelles proportions ces métho- des permettent d'une part, de contenir et de réduire les crues et, d'autre part, de soutenir ou d'augmenter les débits d'eau en période sèche.

2" Rassembler et interpréter les résultats de mesure de taux et de débits de ruissellement dus à des pluies variables en intensité et en quantité; ceci, sur des zones cultivées s'échelonnant du petit bas. sin versant naturel à des superficies de 2.000 à 2.500 hectares.

Le problème à étudier sur les bassins expérimentaux consiste en une étude délaillée et complète du mouvement de l'eau depuis le moment où elle atteint la surface du sol sous forme de précipitation jusqu'à celui où elle quitte le bassin sous forme de courant de surface ou souterrain.

Il comprend l'étude des précipitations, de l'infiltration, de l'évaporation, des suintements, de l'accumulation superficielle ou souterraine et des débits évacués par les cours d'eau de surface. Sous réserve de développements fulurs, le programme générai d'études comprend :

$1^{\circ}$ L’évaluation, grâce à des études expérimentales conduites avec soin, de tous les facteurs qui influent sur l'écoulement de l'eau.

$2^{\circ}$ L'extension aux grands bassins versants des résultats obtenus.

Le but de ce programme est de développer des méthodes permettant d'utiliser les résultats obtenus sur de petits terrains et de petits bassins versants, pour prédire les effets des changements de régime de culture sur de vastes surfaces pouvant présenter une grande variété de sols et de caractéristiques to. pographiques.

(1) Le mot "conservation ", qui peut paraître obscur, verra son sens s'éclairer à la lecture de ce qui suit. II désigne l'ensemble des techniques destinées à préserver les terrains meubles contre toutes actions extérieures tendant à les détériorer et la mise en application de ces techniques Le mot français "préservation " serait mieux adapté, mais nous nous conformerons à l'usage qui a adopté la transcription pure et simple du vocable anglais et nous irons même plus loin en employant, comme l'auteur, l'expression de "conservation des eaux" qui se rapporte aux moyens d'assurer la meilleure utilisation des eaux de toutes sortes circulant ou séjournant tant en surface qu'en profondeur. 
Les resultats obtenus sont de première imporlance, au point de vue hydraulique.. Il sont nécessaires à l'étude économique des ouvrages de proteclion contre les crues et l'érosion et d'autres aménagements hycirauliques. Le manque de données di-
La station de Coshocton est située dans l'est de l'Ohio, à environ 30 kilometres au N.-E. de Colontbus, capitale de cet Etat.

Les premières mesures y commencèrent en 1937. La zone intéressée est caractéristique des zones de

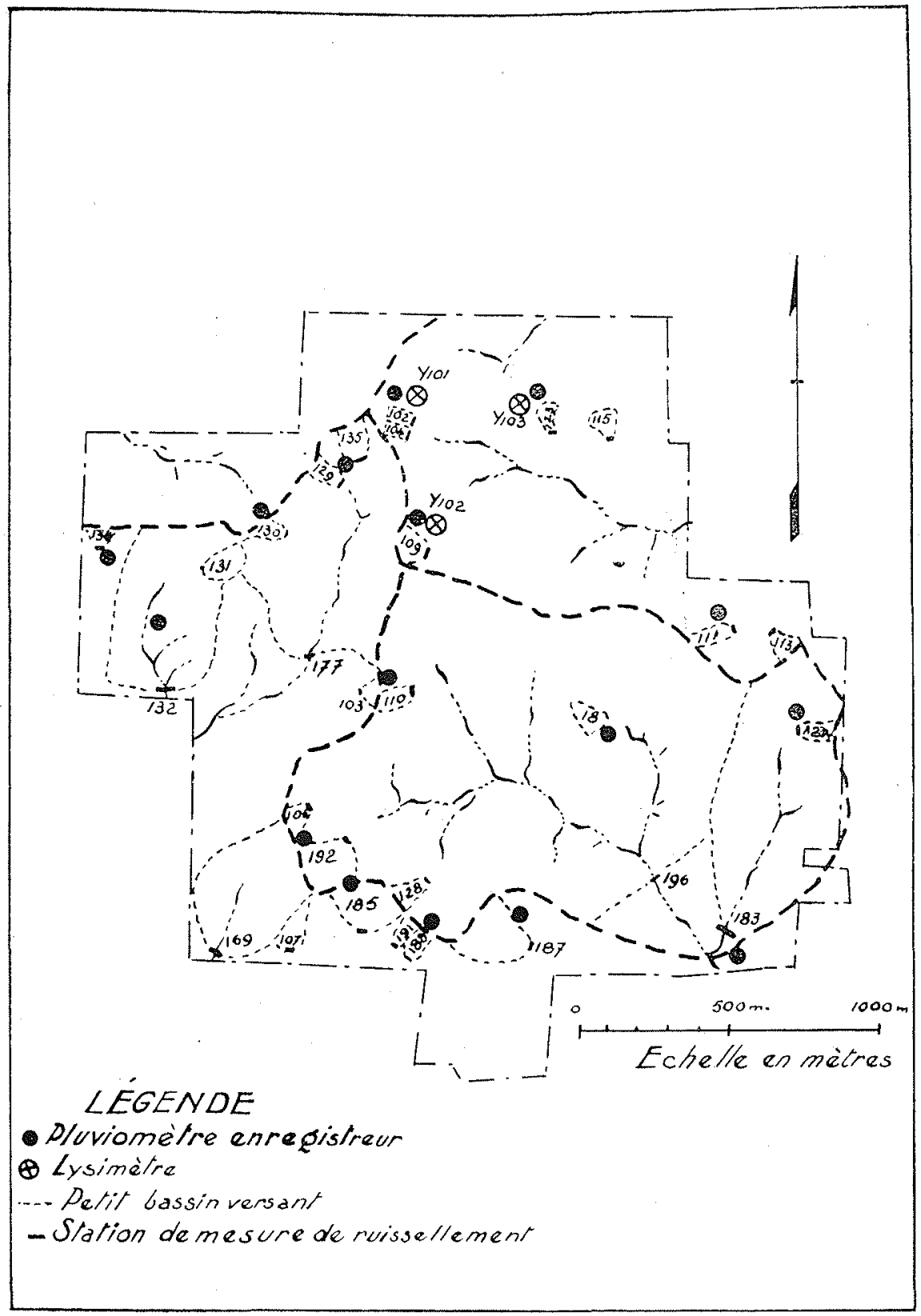

FIG. 1. - BASSINS VERSANTS, PLUVIOMETRES, STATIONS D'ECOULEMENT ET LYSIMETRES SUR LE TERRAIN D'ETAT

gnes de confiance entraine souvent l'insucces complet de tols travaux. Il arrive aussi, plus fréquemment peut-être, que des renseignements insuffisants conduisent à des coefficients de sécurité inutilemeni ćlevés et, par suite, à des prix dont l'importance no se justifie pas. culture de la région naturelle du Nord des Appalaches qui comprend l'Est de l'Ohio, l'Ouest de la Pensylvanie, le Nord-Est du Kentucky et l'Ouest de lix Virginie Occidentale. La zone d'expérience s'étend au Sud des limites de glaciation, à la latitude de $40^{\circ} 22^{\prime}$ Nord et entre les altitudes de 240 et $400 \mathrm{me}$ - 
tres au-dessus du niveau de la mer. Le lype de so! le plus répandu dans cette zone est le limon de Muskigum à provenance de grès et de schistes argileux. Il tombe annuellement dans eette région clima- région où $5 \%$ seulement de la superficie à un: pente inférienre ou égale à $5 \%$ et ou les terrains dont la pente dépasse $20 \%$ représentent environ 30 \% de la surface. La longueur et l'inclinaison des

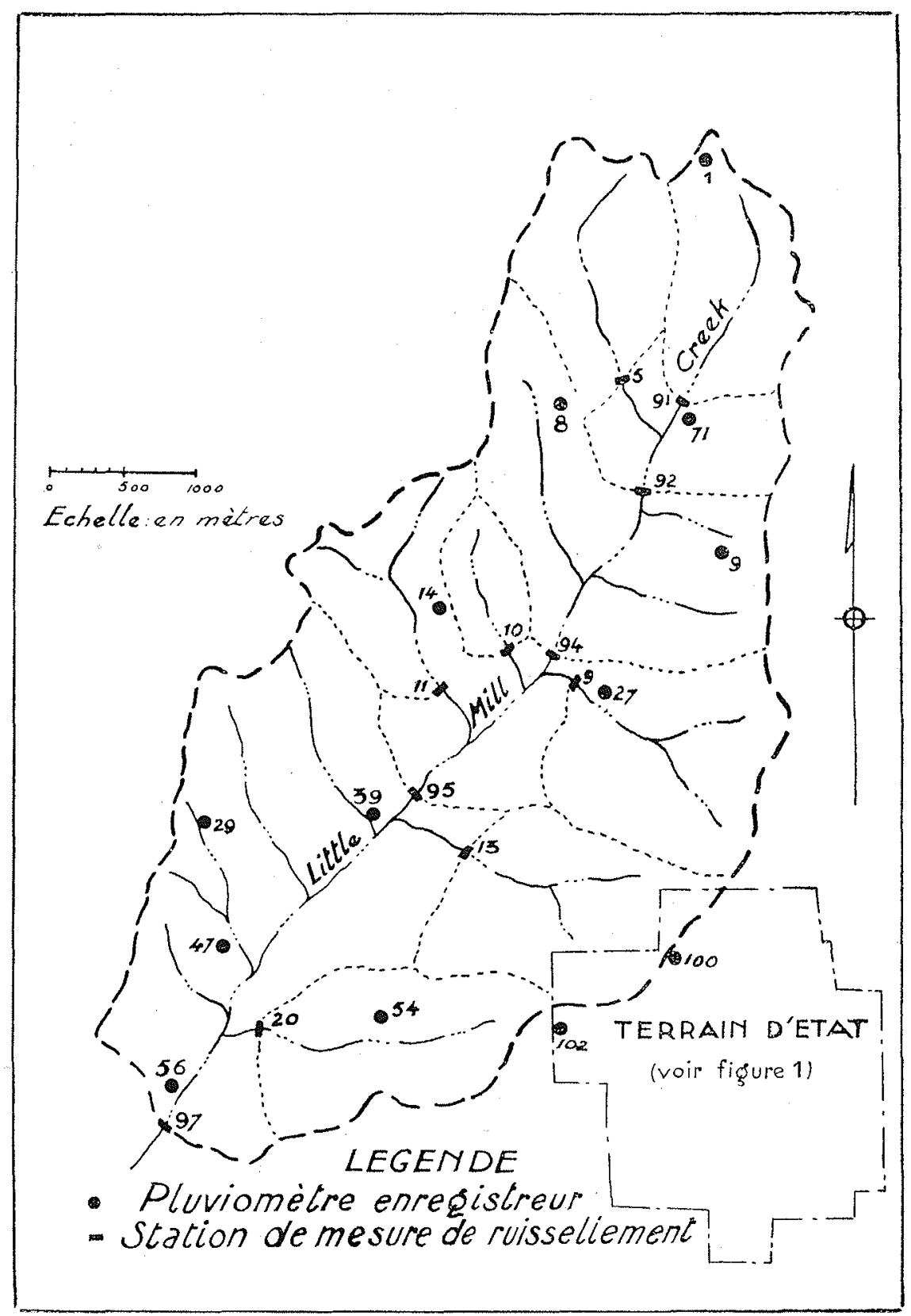

FIG. 2. -.. BASSINS VERSANTS, PLUVIOMETRES ET STATIONS D'ECOULEMENT SUR TERRAINS PRIVES

tique, une moyenne d'environ $97 \mathrm{~mm}$. de pluie. Les mois de plus fortes précipitations coïncident avee la saison de moindre protection du sol. Il en est fréquemment résulté pendant de nombreuses années, de forts ruissellements et de sérieuses érosions du sol. L'érosion est un problème sérieux dans cette pentes influent fortement sur l'erosion. Pour u: accroissement de pente de $100 \%$, lérosion ausmente de 150 à $200 \%$.

L'équipement de la zone d'expérience est indiques sur les figures 1 et 2. La figure 1 montre le terrain de 405 hectares contrôlé par le Gouvernement des 
TABLEAU 1

DESCRIPTION DU BASSIN VERSANT

\begin{tabular}{|c|c|c|c|c|c|c|c|c|}
\hline \multirow[b]{2}{*}{$\begin{array}{l}\text { Bassin } \\
\text { versant } \\
\text { No }^{0}\end{array}$} & \multirow[b]{2}{*}{$\begin{array}{c}\text { Superlicie } \\
\text { drainee } \\
\text { bect. }\end{array}$} & \multirow[b]{2}{*}{$\begin{array}{c}\text { Dèbut } \\
\text { de l'enregistrement }\end{array}$} & \multirow[b]{2}{*}{$\begin{array}{c}\text { Type de lat station } \\
\text { de mesure d'ecoulement }\end{array}$} & \multirow[b]{2}{*}{$\begin{array}{l}\text { Pente } \\
\text { moyenne } \\
\text { du sol \% }\end{array}$} & \multicolumn{4}{|c|}{ Surface drainée en } \\
\hline & & & & & Bois $\%$ & $\begin{array}{c}\text { Pitturige } \\
\text { oll } \\
\text { pralries } \\
\%\end{array}$ & $\begin{array}{c}\text { Cultures } \\
\text { (sauf prairies } \\
\text { d'assolement) } \\
\%\end{array}$ & $\begin{array}{c}\text { Divers } \\
\%\end{array}$ \\
\hline $9 !$ & 119 & Novembre $1930^{\circ}$ & $M$ & & 18 & 64 & 12 & 6 \\
\hline 92 & 372 & SEPTEMbRe 1936 & M & & 21 & 59 & 46 & 4 \\
\hline 94 & 614 & FÉVRIER 1937 & M & & 24 & 57 & 15 & 4 \\
\hline 98 & 1.040 & JANVIER 1937 & M & & 26 & 55 & 15 & 4 \\
\hline 97 & 1.852 & JANVIER 1937 & $\mathbf{M}$ & & 26 & 55 & 15 & 4 \\
\hline 5 & $1: 1$ & MARS 1937 & M & & 22 & 50 & 23 & 5 \\
\hline 9 & 153 & Mars 1937 & $\mathrm{M}$ & 20,0 & 45 & 40 & 12 & 3 \\
\hline 10 & 49 & Aout 1937 & D.S.P. $-4,5$ & - & 25 & 48 & 21 & 6 \\
\hline 11 & 118 & Mars 1937 & DT & - & 31 & 55 & 12 & 2 \\
\hline 13 & 178 & Aovi 1937 & $\mathrm{M}$ & & 45 & 43 & 9 & 3 \\
\hline 20 & 151 & MARS 1937 & M & & 22 & 57 & 18 & 3 \\
\hline 169 & 11,7 & JANVIER 1940 & D.S.P. $-1,2$ & 15,7 & 7 & 44 & 40 & 9 \\
\hline 172 & 17,6 & JANVIER 1939 & D.S.P. $-1,2$ & 22,8 & 99 & 0 & 0 & 1 \\
\hline 177 & $3(j, 6$ & DÈCEMBRE 1939 & D.S.P. $-2,4$ & 15,7 & 11 & .36 & 42 & 11 \\
\hline 183 & 30,1 & FÉVRIER 1938 & D.S.P. $-2,4$ & 14,8 & 14 & 50 & 35 & 1 \\
\hline 196 & 122,5 & AvriL 1937 & $\mathrm{M}$ & 14 & 26 & $4 x$ & 22 & 8 \\
\hline 102 & 0,5 & MARS 1937 & S.P. $-0,3$ & 18,2 & 0 & 100 & 0 & 0 \\
\hline 103 & 0,24 & Mars 1939 & $\mathrm{H}-0,6$ & 11,3 & 0 & 0 & $100(t)$ & 0 \\
\hline 104 & 0,54 & MaRS 1937 & S.P. - 0,3 & 20,7 & 0 & 100 & 0 & 0 \\
\hline 106 & 0,63 & MARS 1939 & $\mathrm{H} \cdot 0,75$ & 14,3 & 0 & 100 & 0 & 0 \\
\hline 107 & 1,05 & JUILLET 1939 & $\mathrm{H}-0,6$ & 15,3 & 100 & 0 & 0 & 0 \\
\hline 109 & 0,44 & JUILLET 1938 & $\mathrm{H}-0,9$ & 12,7 & 0 & 0 & $100(-1)$ & 0 \\
\hline 110 & 0,51 & MARS 1939 & $\mathrm{H}-0,75$ & 13,0 & 0 & 0 & 100 & 0 \\
\hline 111 & 0,48 & Aout 1939 & $\mathrm{H}-0,75$ & 7,5 & 0 & 1) & $100(+)$ & 0 \\
\hline 113 & 0,59 & Aout 1939 & $\mathrm{H}-0,75$ & 9,3 & 0 & 0 & $100(+)$ & 0 \\
\hline 115 & 0,65 & DÉcEMbRe 1938 & $\mathrm{H}-0,75$ & 6,6 & 0 & 0 & 100 & 0 \\
\hline 118 & 0,79 & Aout 1939 & $\mathrm{H}-0,9$ & 9,6 & 0 & 0 & 100 & 0 \\
\hline 121 & 0,58 & MARS 1939 & $\mathrm{H} \cdot 0,75$ & 15,8 & 0 & 0 & $100(+)$ & 0 \\
\hline 123 & 0,55 & DÉCEMBRE 1938 & $\mathrm{H}-0,75$ & 6,8 & 0 & 0 & $100(+)$ & 0 \\
\hline 124 & 0,84 & Aout 1939 & $\mathrm{H}-0,75$ & 12,9 & 0 & 100 & 0 & 0 \\
\hline 128 & 0,90 & JUIN 1939 & $\mathrm{H}-0,9$ & 13,6 & 0 & 0 & 100 & 0 \\
\hline 129 & 1,0 & MaRS 1938 & S.P. $-0,3$ & 18,6 & 0 & 100 & 0 & 0 \\
\hline 130 & 0,65 & Avril 1938 & $\mathrm{~S}, \mathrm{P},-0,22$ & 21,7 & 0 & 100 & 0 & 0 \\
\hline 131 & 0,90 & MAI 1938 & S.P. $-0,15$ & 21,6 & 100 & 0 & 0 & 0 \\
\hline $13 t$ & $0,3 \pi$ & MAI 1938 & S.P. $-0,15$ & 23,2 & 100 & 0 & 0 & 0 \\
\hline 135 & 1,09 & MaRS 1938 & S.P. $-0,3$ & 15,3 & 0 & 100 & 0 & 0 \\
\hline 185 & 2,78 & AouT 1939 & $\mathrm{H}-1,35$ & 13,6 & 0 & 0 & 100 & 0 \\
\hline 187 & 2,91 & AouT 1939 & $\mathrm{HI}-1,35$ & 15,5 & 0 & 0 & 100 & 0 \\
\hline 188 & 0,83 & JUILLET 1939 & $\mathrm{H}-0,9$ & 9,2 & 0 & 0 & 100 & 0 \\
\hline 191 & $0,4 t$ & AouT 1939 & $\mathrm{H}=0,45$ & 0,4 & 0 & 0 & 100 & 0 \\
\hline 142 & 3,18 & AouT 1939 & $H-1,35$ & 15,8 & 0 & 0 & 150 & 0 \\
\hline
\end{tabular}

M - Station de moulinets avec section de jaugeage aménagée.

S.P.I. - seuil Parshall (canal Venturi) de $1 \mathrm{~m}$. de large à l'étranglement.

D.S.P. 5 - double seuil Parshall comportant un déversoir principal de $5 \mathrm{~m}$. de large à l'étranglement et un petit canal complémentaire pour la mesure des faibles débits

H I. - canal de mesure de $1 \mathrm{~m}$. de profondeur.

D. T. - déversoir triangulaire à large seuil. Pente des parois $: 5: 1$

$(+)$ - zones où sont appliquées les méthodes de culture perfectionnées. 
Etats-Unis. C'esi là que se font les études de détail, sur ces petits bassins ne dépassant pas 3 ha. 25 (tableau 1). Sont actuellement en observation : une

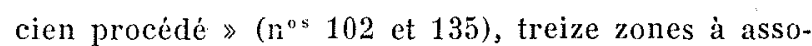
lement de quatre ans : une récolte par an : maïs, blé, foin $\left(n^{\circ s} 103,106,109,110,113,115,118,121,123\right.$,

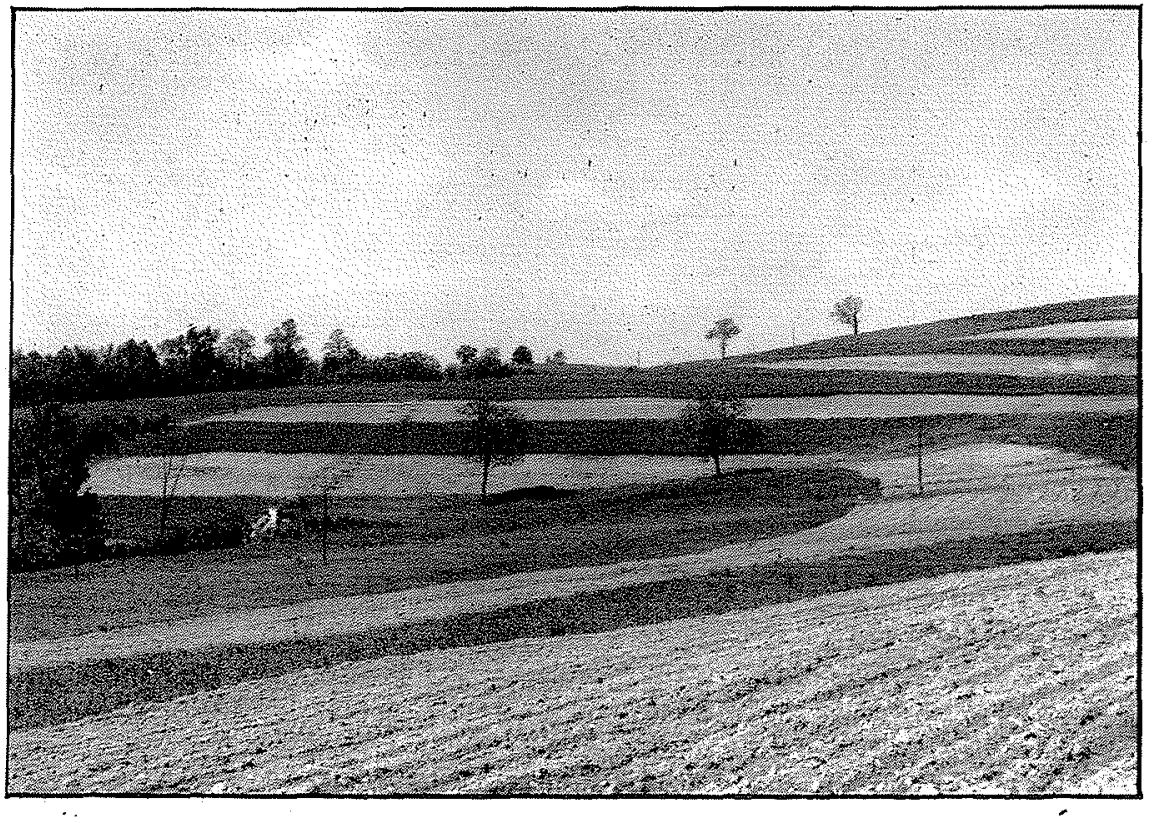

FIG. 3. - BASSIN VERSANT CULTIVE PAR BANDES

CEREALES SEMEES SUR LES BANDES LABOUREES, LUZERNES ET HERBES FOURRAGERES SUR LES BANDES DE GAZON

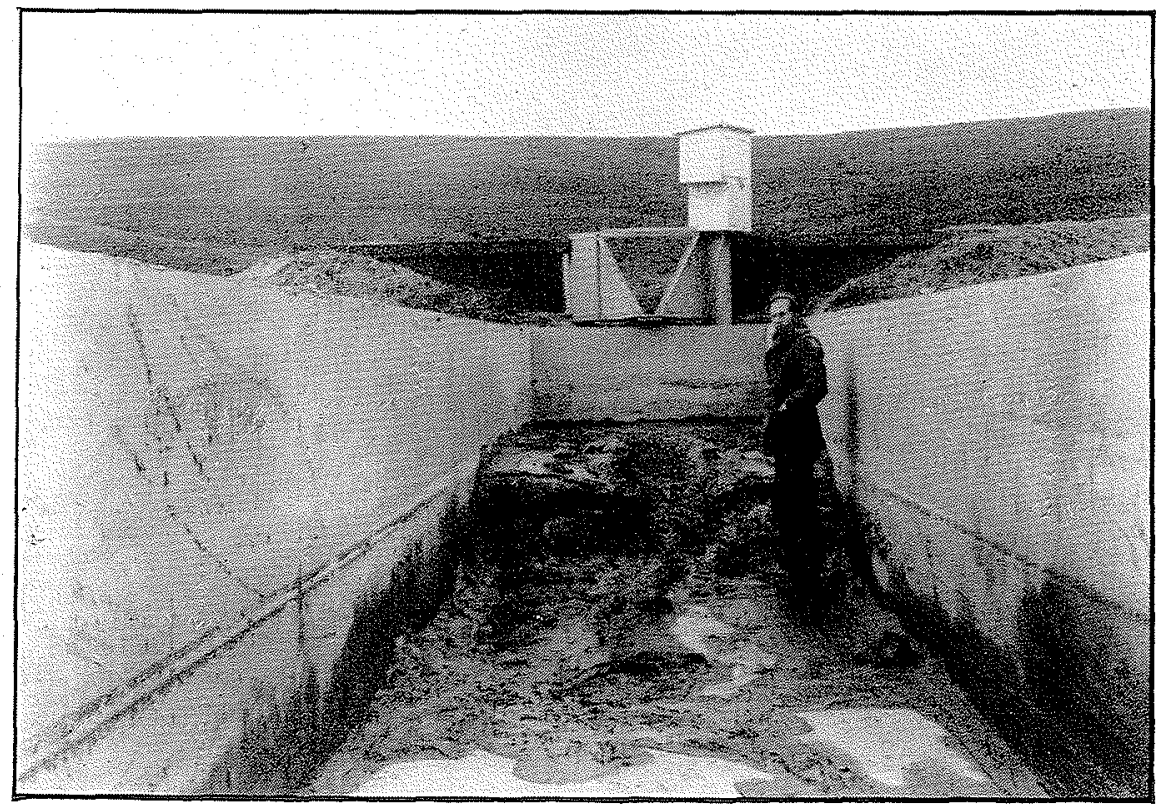

FIG. 4 -.. CANAL (TYPE H) POUR LA MESURE DU RUISSELLEMENT

ET BAC A ARGILE POUR L'EROSION DU SOL SUR UN BASSIN VERSANT DE 0,8 HECTARE.

zone de forêts à maturité ( $n^{\circ} 131$ ), deux zones reboisées (n 107 et 134$)$, une zone de prairie permanente $\left(\mathrm{n}^{\circ} \mathrm{130}\right)$, deux zones de pâturage « perfectionné »( $n^{\text {os }} 104$ et 129$)$, deux zones de pâturage « an-
128, 188, 191 et 192), enfin quatre zones cultivées par bandes ( $\left.\mathrm{n}^{04} 111,124,185,187\right)$.

Parmi les terrains à assolement, il y en a chaque année une paire cultivée en blé, une paire en maïs, 
une paire en prairies de première année et une paire en prairies de seconde année. L'un des ter. rains de chaque paire est cultivé suivant les méthodes perfectionnées telles que la culture en lignes de niveau, et lapplication intensive d'engrais. L'autre est cultivé en sillons rectilignes et avec moins d'en-
$21 \mathrm{~m}$. de largeur, les unes en gazon, les autres labourées.

On effectue des mesures de précipitations, de ruissellements, d'érosion et d'abondance de récoltes sur chacune de ces zones. Les taux et sommes de ruissellements sont déterminés au moyen de l'instal.

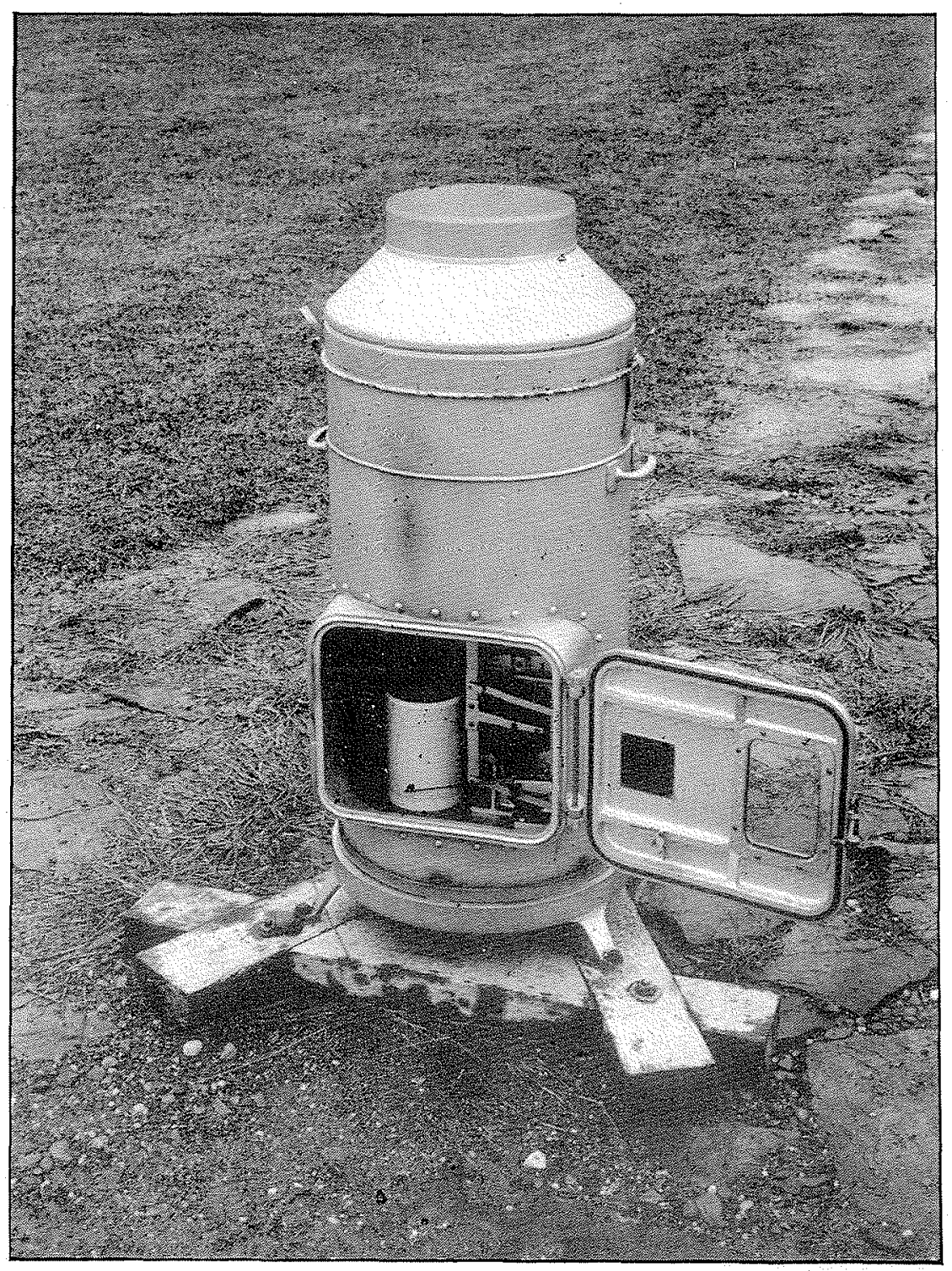

FIG. 5. -- PLUVIOMETRE-ENREGISTREUR, TYPE FERGUSSON

grais. Avant 1941, tous étaient traités de la même façon. Les terrains cultivés par bandes sont à deux cultures : foin et céréales. Une bande sur deux est en céréales et ces bandes sont séparées par une bande de gazon généralement récolté comme fourrage. Toutes les bandes sont d'égale largeur et suivent les lignes de niveat. La figure 3 en montre de lation représentée sur la figure 4. Cette photographie montre un canal en $\mathrm{H}$ avec un abri pour le limnimètre enregistreur automatique, destiné à la mesure précise du ruissellement. La capacité en béton du premier plan sert à mesurer la quantité de sol perdue. On remarque le ravinement du bassin versant, au-dessus du canal. Liappareillage de mesure du 
ruissellement est décrit par Harrold et Krimgold (1).

Les débits instantanés et cumulés des précipita. tions sont déterminés au pluviomètre enregistreur à jesée, représenté sur la figure 5. L'échelle des temps est telle que l'on puisse comnaître les écarts de débit d'une minute à l'autre. La connaissance du débil des précipitations pendant des périodes de moins de cinq minutes est nécessaire à l'analyse de la relation les unes ou denx semaines pendant la saison do croissance des plantes, moins souvent pendant le reste de I'année. Conme le système de racines de Ia plupart des plantes est limité aux profondeurs de 0 à 1 mètre, la prise d'échantillons d'humidité n’a pas été poussée plus profondément. Un graphique montrant l'allure générale des variations d'humidité du sol pendant l'année 1940, est représenté

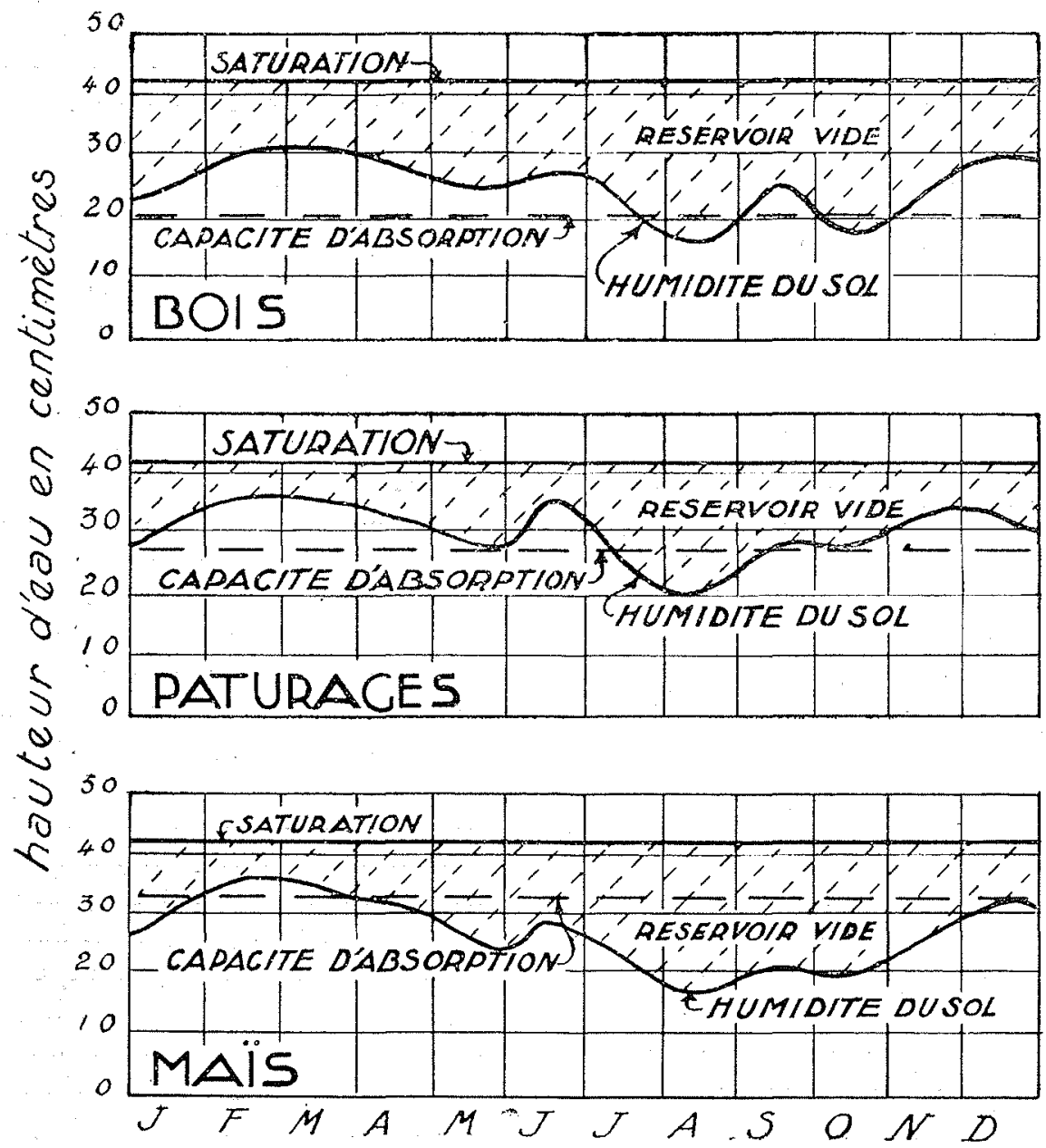

FIG. 6. -- COURBES GENERALES D'HUMIDITE DU SOL DANS LA TRANCHE DE O A I M. SOLS DE LIMON ARGILEUX DE MUSKINGUM COUVERT DE BOIS; PATURAGES, GAZON ET MAIS, EN 1940

précipitation-ruissellement, pour les bassins de drainage de un hectare et moins.

Sur cinq bassins choisis (deux en culture, un en bois et deux en pâturage) on prélève, pour déterminer l'humidité, des échantillons de sol à des profondeurs de 0 à $20 \mathrm{~cm}$., 20 à $35 \mathrm{~cm}$., 55 à $70 \mathrm{~cm}$. et $85 \mathrm{~cm}$. à 1 mètre. Ces prëlèvements sont faits toutes

(1) "Devices for Measuring Rates and Amounts of Runoff", par LL. Harold et D.-B. Krimgold, SCS-TP-51, juillet 1943. U.S. Department of Agriculture. sur la figure 6 . On a donné également pour les forêts, les pâturages et les céréales, la ligne d'humidité à la saturation et la ligne d'humidité que le sol est capable de retenir contre l'action de la pesanteur (capacité d'absorption). Pendant les périodes où l'humidité dépasse la capacité d'absorption, l'eau s'infiltre vers la nappe souterraine.

L'eau qui descend au-dessous de la zone des racines contribue généralement au débit des sources et à l'alimentation en eau souterraine. La mesure 
de ces débits se fait au lysimètres Y 101, Y 102 et Y 103 (fig. 1). La construction et le fonctionnement de ces lysimètres sont suffisamment décrits par Kohnke (1).

L'enregistrement du poids de trois des onze boîtes du lysimètre donne Ies quantités d'eau provenant de l'évaporation à la surface du sol et de la transpiration des plantes. On peut mesurer, à la fois, avec ces lysimètres monoblocs à pesée, les quanti. tés globales et les débits instantanés d'eau. Ainsi, on analyse le mouvement de l'eau depuis le moment où elle atteint le sol sous forme de pluie, jusqu'au moment où elle quitte la zone étudiée par écoulement superficiel ou souterrain. La figure 7 donne des exemples de graphiques des diverses formes do répartition de l'eau de précipitation. Ils ont été établis au lysimètre Y 102 pour l'année 1944.

En plus des bassins versants de moins de 3 ha. 25 , en monoculture, il y a un certain nombre de bassins versants de diverses superficies allant de 12 à 1.850 ha. A l'exception du bassin boisé de 18 ha. n"172, ils portent tous une végétation variée : cultures, pâturages, prairies, bois, etc...; le tableau 1 donne les pourcentages en superficie de ces divers emplois pour chaque zone. Il est à noter que les stations de mesure de ruissellement pour des zones de 50 ha. et moins, sont d'un type étalonné au laboratoire. A cet effet, on a surtout utilisé le canal Parshall. Pour les zones dépassant 120 ha. environ, on a construit des seuils de mesure permanents pour la mesure des faibles débits et l'on s'est servi do moulinets pour établir la relation hauteur-débit. On aurait utilisé, dans tous les cas, des canaux tarés à l'avance s'il avait été possible de les construire à bon marché pour les gros débits. Il est extrêmement difficile d'effectuer des mesures au moulinet, même pour les zones les plus étendues, car les crues croissent et décroissent très rapidement.

Les canaux Parshall (fig. 8) ont un radier plat, de sorte qu'ils ne sont pas adaptés à la mesure précise des petits débits. Ordinairement, ils ne sont pas tarés pour des hauteurs d'eau inférieures à $6 \mathrm{~cm}$. Pour mesurer ces faibles débits, on utilise de petits canaux complémentaires; les débits inférieurs au minimum mesurable au canal principal passent par une ouverture latérale pratiquée dans la section do restitution du grand canal et sont dirigés vers un petit canal où la hauteur d'eau et le débit peuvent être mesurés avec précision. Deux limnimètres enre-

(1) "A Surwey and Discussion of Lysimeters and Bibliography on their Construction and Performance $\Rightarrow$ by Helmut Kohnke, F. $-R$ Dreibeldis and J.-M. Davidson, U.S. Department of Agriculture, Misc. Bul., no 372 , mai 1940. gistreurs sont en service, l'un pour la mesure des hauteurs d'eau dans le grand canal Parshall, l'autre dans le petit. On obtient ainsi des enregistrements précis du débit pendant toute la durée de chaque période de crue et pendant quelques périodes de suintement de l'eau dans le sol.

Deux des bassins versants à couverture mixte restent cultivés suivant la pratique ancienne ou nonperfectionnée. Les autres zones à couverture mixle furent mises en observation pendant quelques an. nées avec les vieilles méthodes de culture, après quoi on commença à appliquer les méthodes de « conservation », mais il faudra encore plusieurs années avant que tous les terrains de chaque zonc soient exploités ainsi. Le bassin non-perfectionné do 30 ha. $n^{\circ} 183$ sera comparé au bassin versant perfectionné de 30 ha. 5 no 177. Les enregistrements des quantités et des débits de ruissellement seront les principales bases de comparaison. De même, le bassin non-perfectionné de 123 ha. $n^{\circ} 196$ sera comparé aux bassins perfectionnés $n^{\circ 5} 5,9,11,12,20$ et 91 .

On étudiera l'effet des procédés de conservation sur les petits bassins à monoculture et sur le bassin à couverture mixte décrit plus haut. Les enregistrements obtenus sur des zones plus grandes $n^{\circ *} 92$, 94, 95 et 97 aideront à déterminer les effets de exs méthodes de culture sur de plus grands bassins.

Il est nécessaire, pour l'étude hydraulique de nombreux ouvrages, de connaître les valeurs des maxima de ruissellement en fonction des dimensions des bassins versants et pour les différentes possibilités de répétition de pluies, On possède, en général, ces données pour les plus grands bassins des Etats-Unis, mais on connât très peu de chose sur les débit: à prévoir sur des bassins inférieurs à $25 \mathrm{~km}^{2}$. Les résultats fournis par notre Station de recherche contribueront à combler ce vide.

Grâce à la variété des bassins, nous pourrons étudier les effets sur la surface drainée des temps de ruissellement, des pointes de crues, de la forme des courbes hydrographiques et de l'accumulation dans les cours d'eau. On peut calculer les fréquences des crues à partir d'enregistrement englobant une courte période el étendre les résultats aux fréquences plus faibles (2), mais on ne peut accorder une entière confiance aux chiffres déterminés par une extrapolation de ce genre. Par exemple, on a analysé un enregistrement sur six années et établi une relation entre les maxima de crues et leur fréquence. La

(2) «Flow from Drainage Basins Determined by Short-term Records" by Llloyd-L. Harold, Proc. Amer. Soc. C. E., avril 1945. 
courbe obtenue fut étendue à une période de cinquante ans et l'on en a déduit une crue cinquantenaire de $17 \mathrm{~mm} .2$ par heure. Une étude fut entreprise sur la période de six ans, pour déterminer si
Or, un an après, un orage survint qui provoqu:i une crue environ trois fois plus forte que la valeur cinquantenaire prévue. Les conditions d'humidilí du sol étaient sensiblement les mêmes que celles qui

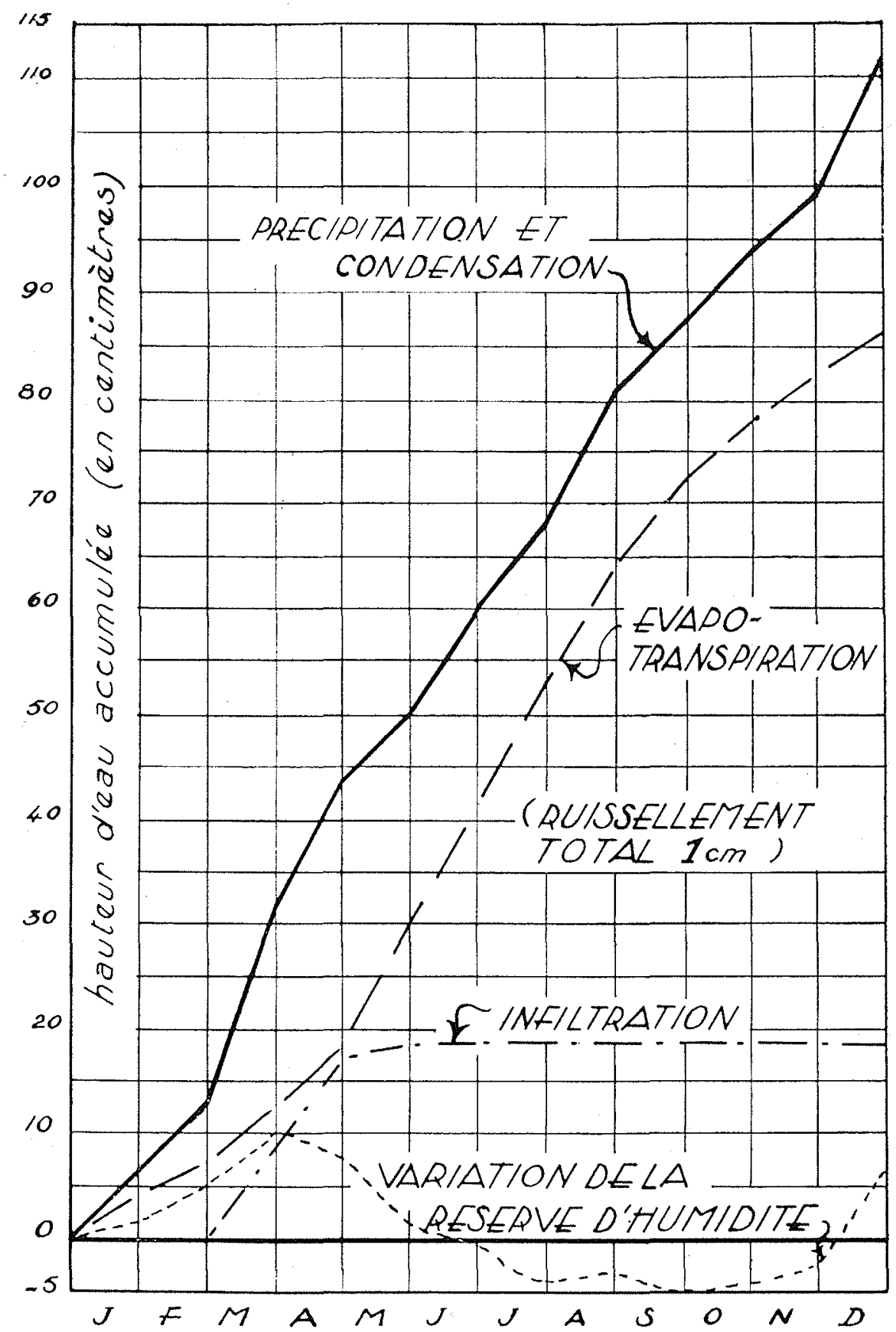

FIG. 7. -- AUGMENTATION ET DIMINUTION DE L'HUMIDITE DU SOL A.CCUMULEE AU LYSIMETRE EN PRAIRIE Y 102 C, 1944

elle était spécialement humide, sèche ou normale. On en a conclu qu'elle était légèrement plus humide que la normale et que la courbe obtenue pouvait. être utilisée en toute sécurité. s'étaient présentées plusieurs fois pendant la périods. de six ans; les débits maxima de pluie pendant ce dernier orage n'avaient pas été plus élevés que ceux des plus forts orages de la période de six ans; c'est 
une coincidence de forts débits de précipitation avec une humidité déjà très grande du sol qui produlsit ce débit de ruissellement anormalement fort.

On peut se demander au bout de combien de temps il faut s'allendre à voir se reproduire une crue de cette amplitude.

Evidemment, il faut avoir un enregistrement sur sous une forme telle qu'ils puissent être utilisés rapidement pour toute espèce d'analyse, qu'elle concerne les valeurs globales, les débits instantanés, les répartitions dans le temps ou toute étude similaire. Avant que la seconde guerre mondiale n'englobe les Elats-Unis d'Amérique, un programme avait été établi pour la publication des résultats hydrologiques

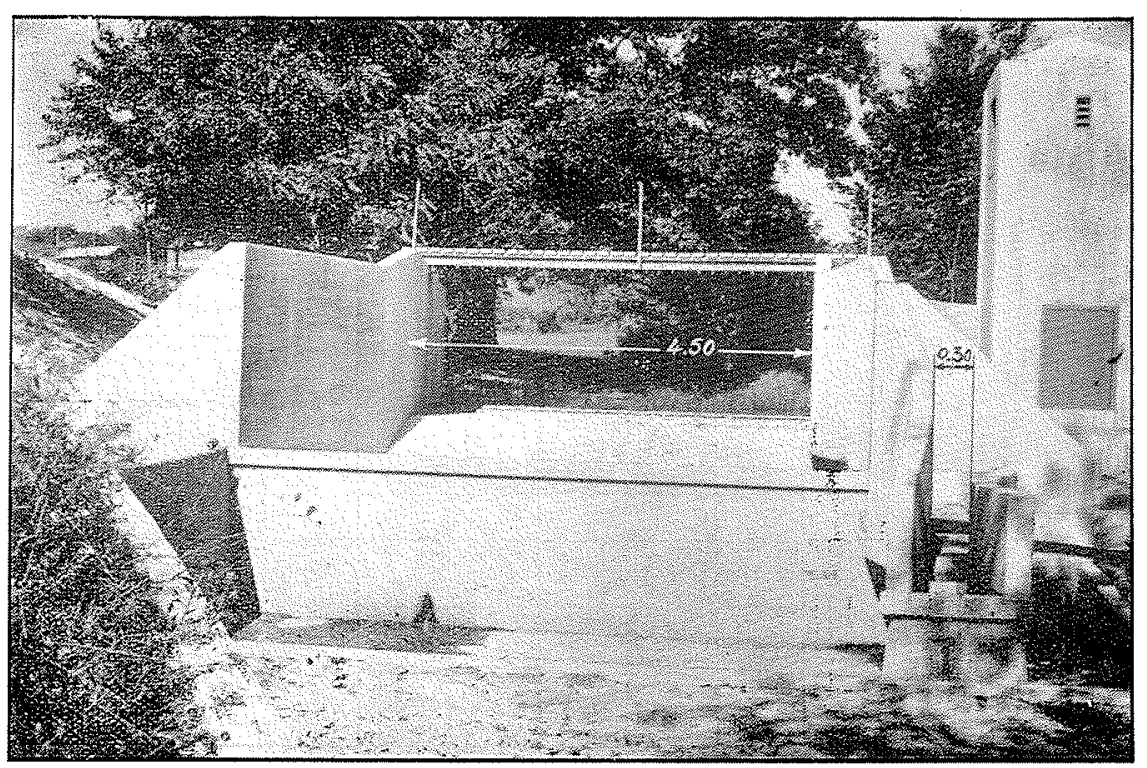

FIG. 8. -- CANAL PARSHALL

une période plus longue, avant de pouroir répondre de façon satisfaisante à cette question. On prévoit de poursuive ces études hydrologiques pendant une période de vingt ans ou plus, pour que leurs résuitats puissent être utilisés en toute confiance.

Les enregistrements locaux sont constamment compilés et rassemblés en tableaux. Ils sont mis fondamentaux, provenant de nos bassins ou d'autres. Ce programme fut interrompu et n'a pas encore été rétabli. On envisageait de publier les résultats fondamentaux chaque année et, tous les trois ou cinq ans, des bulletins spéciaux sur les découvertes et l'analyse des résultats. Peut-être ce programme serat-il repris dans un proche avenir. 